\title{
Critical Behavior of Heisenberg Chains Coupled to Phonons
}

\author{
Rainer W. Kühne \\ Vorm Holz 4, 42119 Wuppertal, Germany
}

We present a quantum Monte Carlo study for the isotropic antiferromagnetic Heisenberg chain coupled to Einstein phonons. By investigating the behavior of the correlation length $\xi$ we find the critical exponent $\nu$ to depend on both the phonon frequency $\Omega$ and the spin-phonon coupling constant $g$. The relation is $\xi / a=(1.215 \pm 0.020)(1+$ $\left.g^{2} J / \Omega\right)(T / J)^{-\nu}$, where $\nu=1-0.5|g|^{3 / 2} J / \Omega$. Hence, these systems change the universality class continuously with the spin-phonon coupling.

PACS numbers: $63.20 . \mathrm{Kr}, 75.10 . \mathrm{Jm}, 75.40 . \mathrm{Mg}, 75.50 . \mathrm{Ee}$

The critical behavior of Ising models and isotropic Heisenberg systems is well-understood. An important question to address is whether the introduction of further degrees of freedom such as disorder and phonons changes the universality class of the transition. The situation for distorted spin systems has been discussed controversially in the literature, that for spin-phonon systems will be examined in this study.

Some evidence has been presented for different critical behavior of distorted and pure spin systems. A numerical transfer matrix study for quenched random bonds in the two-dimensional ferromagnetic Ising model suggested the critical exponent $\nu$ to change with the addition of quenched random defects, $\nu>1$ (Ref. 1), where for the pure two-dimensional Ising model it is $\nu=1$. A Monte Carlo study of the two-dimensional randomly site diluted Ising model argued for concentration dependent critical ipdices, especially $\nu>1$ (Refs. 2 and 3 and discussion 6 6). A combination of the grand ensemble approach and phenomenological renormalization for the two-dimensional spin diluted Ising model suggested a continuous variation of the critical exponents $\gamma$ and $\nu$ with the density of magnetic impurities, fespecting weak universality, i. e. $\eta$ and $\gamma / \nu$ remain fixed $]$.

By contrast, high-temperature series expansions of the two-dimensional random-bond Ising ferromagnet found $\gamma$ to increase steadily with disorder only if the powerlaw $\chi \propto t^{-\gamma}$ (where $\chi$ is the magnetic susceptibility and $\left.t \equiv\left(T-T_{c}\right) / T_{c}\right)$ is assumed. If logarithmic corrections of the form $\chi \propto t^{-\gamma}|\ln t|^{\gamma / 2}$ are taken into account the universality class is not changed 1 . Furthermore, Monte-Carlo simulations of the two-dimensional Ising model with randomly distributed ferromagnetic bonds have shown only the critical exponent $\beta_{d}$ of the defect magnetization to change continuously with the strength of the defect coupling. However, the critical exponent $\nu$ of the random Ising model has been reported to not depend on dilution, i. e. $\nu=1$ (Ref. 9).
Let us turn to antiferromagnetic Heisenberg chains. A complete and exact diagonalization of the pure system has shown the critical exponent $\nu=1.0$ (Ref. 10). Strong spin-phonon coupling is known to generate dimerization 11 17, thus altering the properties of the pure spin-chain. This prompted us to examine whether chains with nonzero spin-phonon coupling enjoy the same critical behavior.

We perform a quantum Monte Carlo simulation of the spin- $1 / 2$ isotropic antiferromagnetic Heisenberg chain coupled to Einstein phonons,

$$
\mathcal{H}=\frac{J}{2} \sum_{l=1}^{N}\left(\vec{\sigma}_{l} \vec{\sigma}_{l+1}-1\right)\left(1+g\left(b_{l}^{\dagger}+b_{l}\right)\right)+\Omega \sum_{l=1}^{N} b_{l}^{\dagger} b_{l},
$$

by using the extended loop algorithm described in Ref. 17. We choose the chain length $N=64$ and the Trotter number $M=80$, because for these values finitesize effects are negligible for temperatures $T \geq 0.2 J$. For all our runs we find a truncation of 50 phonons per site to be sufficient for the temperature range investigated, $0.2 J \leq T \leq J$, the probability for more than 50 phonons per site is less than $10^{-6}$.

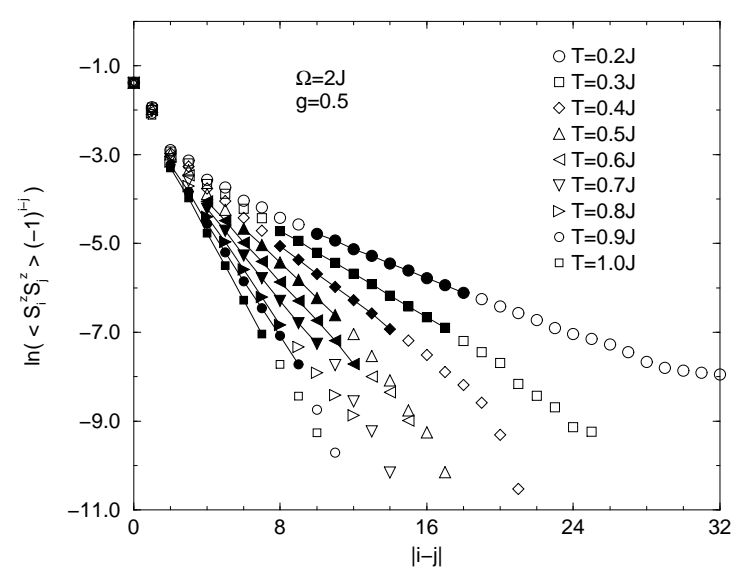

FIG. 1. The logarithm of the correlation function $<S_{i}^{z} S_{j}^{z}>(-1)^{i-j}$ versus $|i-j|$. Black symbols show the region where the correlation function decays exponentially. The correlation length $\xi$ was determined by using the data points of this region only.

We examine the correlation length $\xi$ of the correlation function $G(r)=<S_{i}^{z} S_{j}^{z}>(-1)^{i-j}$, where $S_{i}^{z}$ is the zcomponent of the $i$ th spin. In one-dimensional systems this function decays according to $G(r) \propto \exp (-r / \xi)$ for 
$r \gg \xi$ (Ref. 18), where $r=a|i-j|$ and $a$ denotes the lattice constant. Because of statistical fluctuations quantum Monte Carlo simulations allow the examination of this decay only in a finite region of $r$. A typical result is shown in Fig. 1. The correlation lengths of all the systems we investigated are $a<\xi<7 a$. Therefore the chain length $N$ is (with few exceptions) at least an order of magnitude larger than $\xi$. Hence, finite-size effects are unlikely to alter our results significantly. Furthermore, a simulation with $N=64$ and $M=80$ for the pure Heisenberg chain in the temperature range $0.2 J \leq T \leq J$ yielded $\xi$ within an inaccuracy of $\leq 3 \%$, when compared with the exact data of Ref. 19. This value sets an upper limit on the effects of finite size and finite Trotter number.
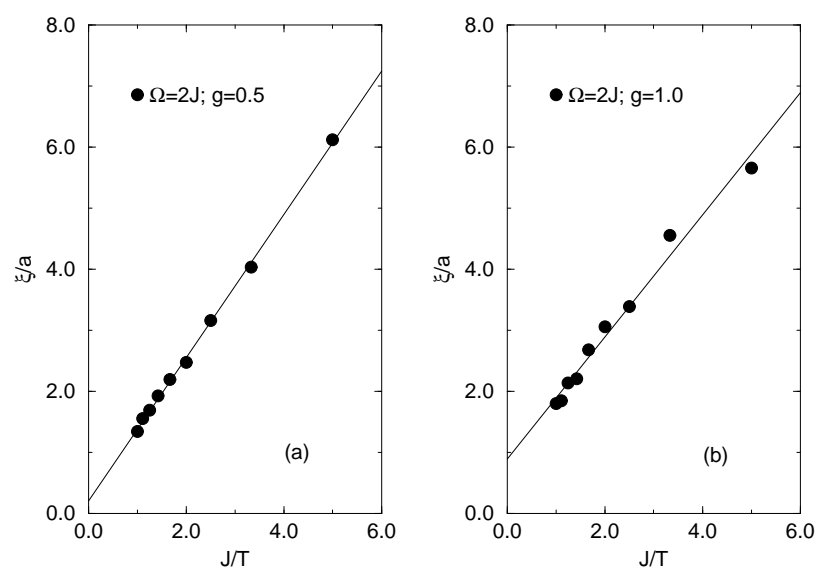

FIG. 2. The correlation length $\xi$ in units of the lattice constant $a$ as a function of the inverse temperature. The solid line is the linear fit. The existence of the offset disproves the suggested proportionality $\xi \propto T^{-1}$.

If spin-phonon chains and pure spin chains enjoy the same critical behavior we would expect $\xi \propto T^{-\nu}$ with $\nu=1$. In Fig. 2 we present $\xi$ as a function of the inverse temperature. The appearance of the offset refutes the suggested proportionality. Hence, spin-phonon chains do not enjoy the same critical behavior as pure spin chains.

In the following we will examine the dependence of the correlation length $\xi$ (in units of the lattice constant $a$ ) on the phonon frequency $\Omega$ and the spin-phonon coupling constant $g$. The formula we would like to determine is $\xi / a=f(g, \Omega)(T / J)^{-\nu(g, \Omega)}$.

We first determine the best fit values of $\nu$ for the proportionality $\xi \propto T^{-\nu}$. Typical curves are shown in Fig. 3 . Afterwards we examine the dependence $\nu=\nu(g, \Omega)$. The proportionality $1-\nu \propto 1 / \Omega$ is shown in Fig. 何, the proportionality $1-\nu \propto g^{3 / 2}$ is shown in Fig. 4 b. By using various sets for $g$ and $\Omega$ we find $1-\nu=0.5|g|^{3 / 2} J / \Omega$ (Fig. 司).

Naturally, this relation is valid only for systems with not too large values of $|g(\Omega)|$. For larger values of $g$ the appearance of dimerization destroys the region of exponential decay and therefore does not allow the definition of the correlation length. This is why we were not able to identify critical exponents $\nu<0.7$ for high values of $|g|^{3 / 2} J / \Omega$.
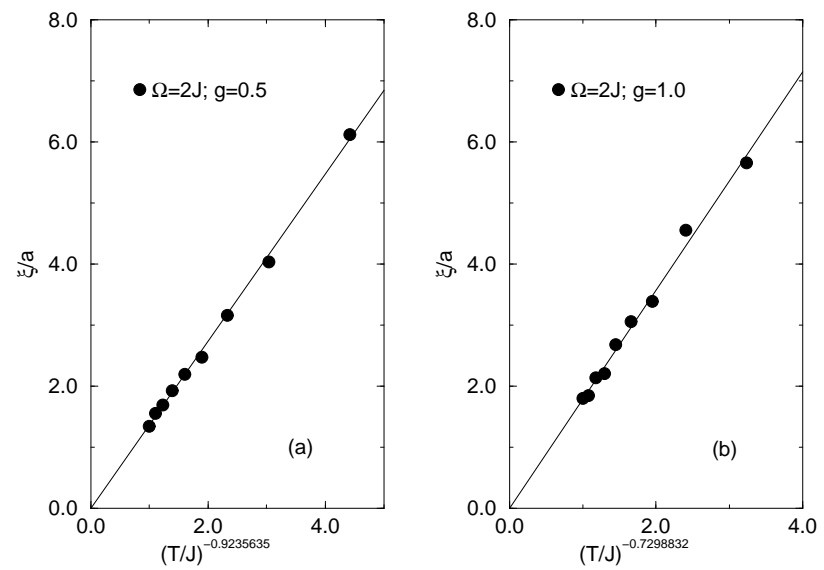

FIG. 3. The correlation length $\xi$ in units of the lattice constant $a$ as a function of the best fit for the critical exponent $\nu$. The solid line is the linear fit.
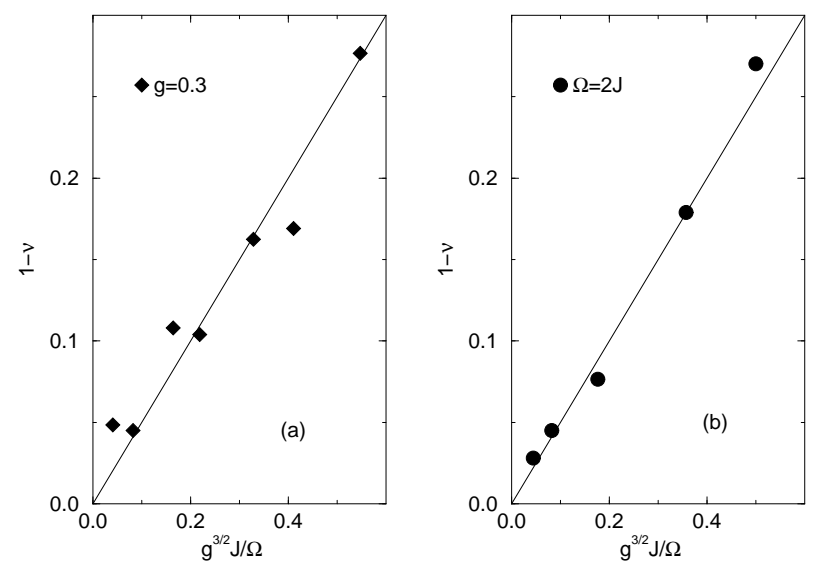

FIG. 4. The deviation of the critical exponent $\nu$ from the Heisenberg value 1 in dependence of (a) $1 / \Omega$ and (b) $g^{3 / 2}$. The solid lines denote the relation $1-\nu=0.5 g^{3 / 2} \mathrm{~J} / \Omega$.

We proceed by plotting $\xi$ as a function of $(T / J)^{-1+0.5|g|^{3 / 2} J / \Omega}$. Typical graphs are shown in Fig. 6. The slope parameter gives the value for $f(g, \Omega)$.

The dependence of $f$ on $\Omega$ is shown in Figs. $7 \mathrm{a}$ and c, that on $g$ in Figs. $7 \mathrm{~b}$ and d. We find $f(g, \Omega)=c(1+$ $\left.g^{2} J / \Omega\right)$. The proportionality constant is $c=1.215 \pm 0.020$ (Fig. 8).

To test our Eq. (2) we present $\xi$ for fixed temperature $T=J$ and various choices of $g$ and $\Omega$ in Fig. 9. For $T=J$ 
this equation simplifies to $\xi / a=f(g, \Omega) \equiv c\left(1+g^{2} J / \Omega\right)$. We prove Eq. (2) to be correct where our best fit yields $c=1.212$. This is in agreement with the exact result for the pure Heisenberg chain, $c_{H}(T=J)=1.203$ (Ref. 19).

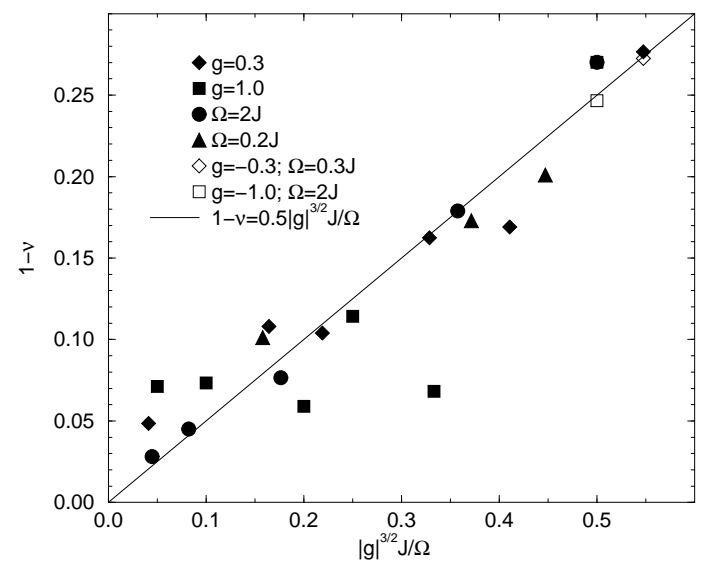

FIG. 5. The deviation of the critical exponent $\nu$ from the Heisenberg value 1 as a function of $|g|^{3 / 2} J / \Omega$.
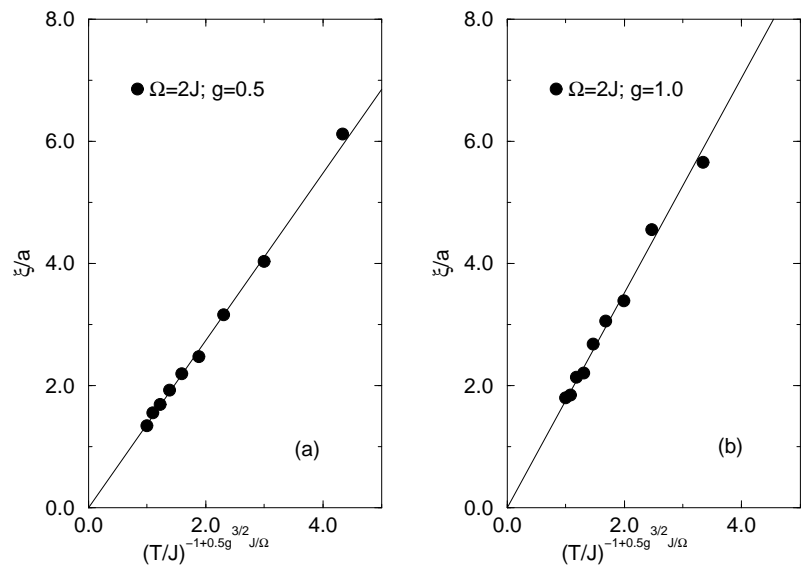

FIG. 6. The correlation length $\xi$ in units of the lattice constant $a$ as a function of $(T / J)^{-\nu}$, where $\nu=1-0.5 g^{3 / 2} J / \Omega$. The solid line is the linear fit.

The correlation lengths of all our runs can be fitted by the proportionality $\xi \propto(T / J)^{-\nu}$ where $\nu=$ $1-0.5|g|^{3 / 2} J / \Omega$ (typical graphs are shown in Fig. 6). Furthermore, for $T=J$ the correlation lengths of all our runs satisfy $\xi / a=c\left(1+g^{2} J / \Omega\right)$ where the proportionality constant $c$ is in very good agreement with the value for the pure Heisenberg chain (Fig. 9). Hence, the exponent $\nu$ we examined is not an effective exponent. Its variation with $g$ and $\Omega$ is real and not a spurious artifact from a non-power-law behavior of the correlation length.
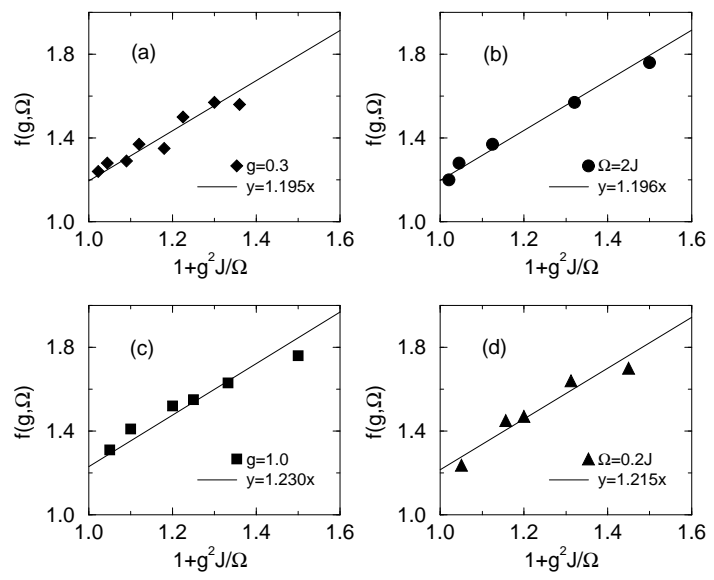

FIG. 7. The factor $f(g, \Omega)$ as (a) a function of $\Omega$, (b) a function of $g$, (c) a function of $\Omega$, and (d) a function of $g$. The solid lines are linear fits. Ideally, they should be identical.

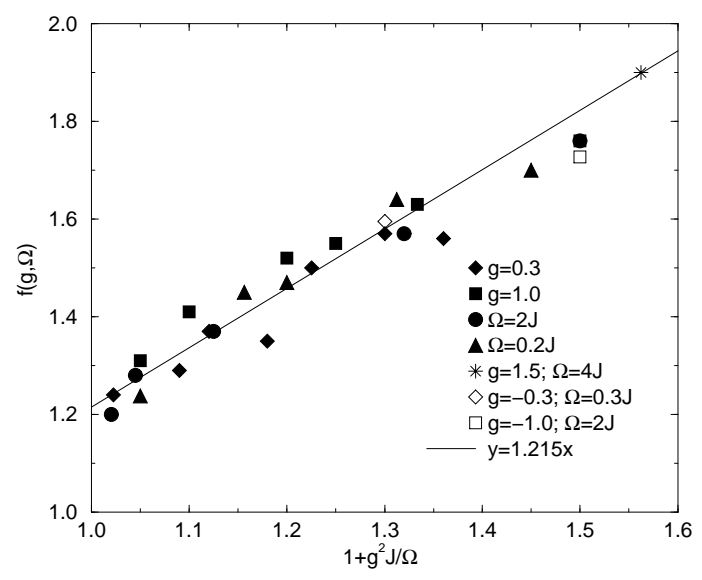

FIG. 8. The factor $f(g, \Omega)$ as a function of both $g$ and $\Omega$. The solid line is the linear fit.

In summary, we found evidence for different critical behavior of the spin- $1 / 2$ isotropic antiferromagnetic Heisenberg chain coupled to Einstein phonons with respect to the pure system. The dependence of the correlation length $\xi$ on the phonon frequency $\Omega$ and the spin-phonon coupling constant $g$ is

$$
\frac{\xi}{a}=(1.215 \pm 0.020)\left(1+\frac{g^{2} J}{\Omega}\right)\left(\frac{T}{J}\right)^{-1+0.5|g|^{3 / 2} J / \Omega},
$$

where $a$ is the lattice constant, $T$ is the temperature, and $J$ is the spin-spin coupling constant. The value $c=1.215 \pm 0.020$ is in accordance with the value found for the pure Heisenberg chain, $c_{H}=1.18 \pm 0.03$, in the 
temperature range $0.2 J \leq T \leq J$ (Ref. 19). In this temperature range $c_{H}$ varies slightly (by $5 \%$; possibly due to logarithmic corrections). From our data sets (cf. Fig. 6) we can infer an upper limit for the variation of $c$ in this temperature range to be $10 \%$.

This result may be of importance for the understanding of materials like $\mathrm{Sr}_{2} \mathrm{CuO}_{3}$ and $\alpha^{\prime}-\mathrm{NaV}_{2} \mathrm{O}_{5}$ whose (hightemperature) dynamics can be described by a nearestneighbor spin-1/2 isotropic antiferromagnetic Heisenberg chain 20.21.

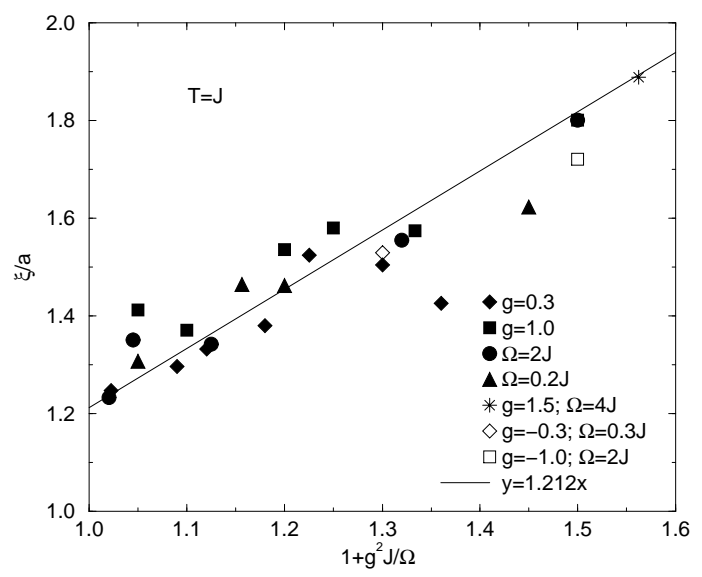

FIG. 9. The correlation length $\xi / a$ as a function of $1+g^{2} J / \Omega$ for fixed temperature $T=J$. The solid line is the linear fit.

${ }^{1}$ M. A. Novotny, Phys. Rev. Lett. 70, 109 (1993).

${ }^{2}$ J.-K. Kim and A. Patrascioiu, Phys. Rev. Lett. 72, 2785 (1994).

3 J.-K. Kim and A. Patrascioiu, Phys. Rev. B 49, 15764 (1994).

${ }^{4}$ W. Selke, Phys. Rev. Lett. 73, 3487 (1994).

${ }^{5}$ K. Ziegler, Phys. Rev. Lett. 73, 3488 (1994).

${ }^{6}$ J.-K. Kim and A. Patrascioiu, Phys. Rev. Lett. 73, 3489 (1994).

${ }^{7}$ R. Kühn, Phys. Rev. Lett. 73, 2268 (1994).

${ }^{8}$ A. Roder, J. Adler, and W. Janke, Phys. Rev. Lett. 80, 4697 (1998).

${ }^{9}$ F. Szalma and F. Igloi, J. Stat. Phys. 95, 759 (1999).

${ }^{10}$ K. Fabricius, U. Löw, K.-H. Mütter, and P. Überholz, Phys. Rev. B 44, 7476 (1991).

${ }^{11}$ A. W. Sandvik, R. R. P. Singh, and D. K. Campbell, Phys. Rev. B 56, 14510 (1997).

12 D. Augier and D. Poilblanc, Eur. Phys. J. B 1, 19 (1998).

${ }^{13}$ D. Augier, D. Poilblanc, E. Sorensen, and I. Affleck, Phys. Rev. B 58, 9110 (1998).
${ }^{14}$ P. Hansen, D. Augier, J. Riera, and D. Poilblanc, Phys. Rev. B 59, 13557 (1999).

${ }^{15}$ R. J. Bursill, R. H. McKenzie, and C. J. Hamer, Phys. Rev. Lett. 83, 408 (1999).

${ }^{16}$ A. W. Sandvik and D. K. Campbell, Phys. Rev. Lett. 83, 195 (1999).

${ }^{17}$ R. W. Kühne and U. Löw, Phys. Rev. B 60, 12125 (1999).

18 J. L. Cardy, Scaling and Renormalization in Statistical Physics (Cambridge Univ. Press, Cambridge, 1996), p. 7.

19 A. Klümper, Eur. Phys. J. B 5, 677 (1998).

${ }^{20}$ M. Takigawa, O. A. Starykh, A. W. Sandvik, and R. R. P. Singh, Phys. Rev. B 56, 13681 (1997).

${ }^{21}$ M. Isobe and Y. Ueda, J. Phys. Soc. Japan 65, 1178 (1996). 